\title{
Fast Discrete Curvelet Decomposition with Gradient Fusion Based Technique for Pansharpening of Multispectral Image
}

\author{
Leelavathi H P, J Prakash
}

\begin{abstract}
The Process of improving the local details of multispectral image using the information captured from different image sensors is called pansharpening. In this paper, the information captured from three different satellite sensors such as synthetic aperture radar (SAR), multispectral (MS) and panchromatic (PAN) of the same scene is combined effectively using the proposed a novel approach. This approach consists of three stages of fusion scheme, where block based wrap fast discrete curvelet decomposition technique is used in the first stage, which results an intermediate PAN image that contain the almost complete spatial details of SAR image and nonadaptive sparse details of the SAR and PAN images. Next the gradient fusion based technique is used to combine the SAR and an intermediate PAN image which retains more information in the PAN-SAR (PS) fused image. Further pansharpening of the MS image is accomplished by using the hybrid technique which is based on injecting the primary and secondary high frequency components into the original MS image. In this algorithm, the high frequency details are extracted by taking difference between the PS and synthesized intensity image, then the extracted high frequency details are modulated by local adaptive and post processing fusion parameter. The experimental outputs reveal that the proposed algorithm results better performance than other techniques by providing more spatial information in multispectral image while preserving the spectral details and has the potential characterize urban areas in a fused image in a better way.

Index terms- Curvelet Transform, Gradient Fusion, Hybrid Pansharpening, Multi-sensor Image Fusion, Multispectral, Panchromatic, Spatial Resolution, Synthetic Aperture Radar.
\end{abstract}

\section{INTRODUCTION}

With the rapid advancement in the earth observing satellites, different remotely sensed imagery such SAR, MS and PAN images having different resolution have been obtained. Each these images are formed by using different image formation mechanism, hence each of these images has its own kind of characteristics and consist of different sort of information. SAR is an active sensor which operates at $\mathrm{C}$ band having its own illumination and observes the earth's surface 24/7 with all weather conditions. In SAR, image formation depends on backscattering mechanism. SAR image has high spatial information in a densely populated area where large numbers of high rise buildings with complex roof and different structures are located sealtogether, because a high backscattering response results from high dielectric properties of various objects.

Revised Manuscript Received on June 15, 2020.

* Correspondence Author

Leelavathi H P*, Associate Professor, Department of ECE, Vivekananda Institute of Technology, Bangalore, hplvkit@gmail.com,

Dr. J Prakash, Prof and Head, Department of ISE, Bangalore Institute of Technology, Bangalore, Karnataka State, India, Jogaiahprakash@gmail.com.

(C) The Authors. Published by Blue Eyes Intelligence Engineering and Sciences Publication (BEIESP). This is an open access article under the CC BY-NC-ND license (http://creativecommons.org/licenses/by-nc-nd/4.0/)
One of the earth observing sensor which captures the earth's surface in multiband and results the multispectral images, typically these sensors are passive sensors require sun's illumination for their operation and cover infrared, visible bands of the EM spectrum by providing less than 15 bands. PAN image is generated by combining the visible bands such as RGB and infrared band of multispectral image that results in single band image. Since PAN image is produced by using the complete light energy in the visible and infrared bands, a huge amount of lunar power collected per pixel that provide more spatial details in the panchromatic image than multispectral image [18]. However, the multispectral image has high spectral information, hence these images have complementary information, hence all these images are usually combined to improve the image visual interpretation and missing information recovery. Several fusion techniques have been proposed to combine complementary details of SAR, multispectral and panchromatic images and resultant fused image finds the significant demands in the remote sensing applications like land monitoring, maritime monitoring emergency management etc. In the past few decades, various multispectral pan sharpening schemes have been proposed to merge the high spatial components of panchromatic image into MS image to increase ground details. The satellite image fusion scheme based on an adaptive pan sharpening with deep self learning network is developed using the algorithm called point spread function (PSF) estimation [1]. This algorithm is used to get the multispectral image blue kernel, further an edge detection based co registration technique is designed for better geometric alignment among the panchromatic (PAN) and multispectral images. Later multispectral image is down sampled using approximated PSF to train the pansharpening network. Finally high resolution MS image is obtained by prediction of the trained deep self learning network using multispectral and panchromatic images. Adaptive intensity -hue-saturation with multi scale guided filter based fusion scheme is proposed to sharpen multispectral image by inserting detail components of the PAN [2]. First upsampled multispectral image is used to obtain the intensity component adaptively, then multi scale guided filter is used to acquire the more detail components of panchromatic image. Fused image is generated by the model based algorithm in which detail components of panchromatic image are inserted into all the bands of an upsampled multispectral image using the injection gains strategy to control the quality of detail components injection.

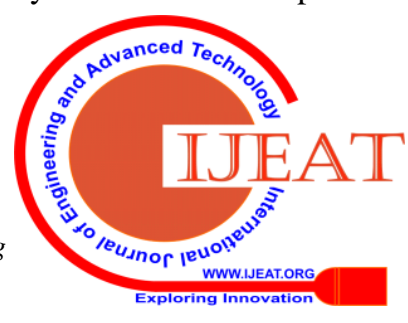

Blue Eyes Intelligence Engineering

\& Sciences Publication

(C) Copyright: All rights reserved. 


\section{Fast Discrete Curvelet Decomposition with Gradient Fusion Based Technique for Pansharpening of Multispectral Image.}

Spatially adaptive intensity- hue -saturation algorithm is developed to mitigate the spectral distortion with injecting the automatically adjusted precise spatial components of panchromatic image into the multispectral image while performing fusion task [3]. In this algorithm first intensity image is generated by selecting the pixels of panchromatic and multispectral image which resembled maximum, the resulting image provides the spectral details of multispectral image rather than providing the high frequency details of panchromatic image. The pixels which cause the frequency distortion are controlled by assigning the lower weight to them, which also prevents the occurrence of redundant spatial components in fused image. The pan sharpening technique based on the component substitution method which uses the local and global correlation coefficients is developed to merge the PAN and MS images [4]. In this algorithm first high resolution adjusted intensity image is generated by partial injection of multispectral bands into panchromatic image, then the histogram matched PAN image is obtained using mean and standard deviation of PAN and multispectral images. Further the fusion parameters are computed using the properties like the statistical ratio of the standard deviation and spatial correlation of multispectral bands and corresponding adjusted intensity images. Finally pan-sharpened multispectral image is obtained by merging multispectral bands with difference image of histogram matched PAN and high resolution adjusted intensity image which is modified by the obtained fusion parameter. Synthetic aperture radar image is combined with the optical image using a fusion scheme which is based on an area based algorithm to enhance the acuity of image [5]. In this scheme first, SAR and optical images are geometrically aligned using co registration process, then gray level co-occurrence matrix image analysis is used to obtain the entropy texture feature of SAR image. Further split and merge segmentation is used to segment texture image of SAR into homogeneous and heterogeneous areas, then homogeneous areas of SAR is injected into the PAN image using the feature selection rule. Finally a multispectral image is pan sharpened using the hybrid pan sharpening approach. A new fusion scheme based on the IHS technique with adjusted fusion parameter is proposed to merge the satellite images [6]. In this scheme first, IHS approach is applied to the MS image to convert RGB bands into IHS data bands, then new intensity image which consists of spatial details of PAN and high frequency details of MS image is generated using a trade-off fusion parameter to balance the spatial and frequency distortion in the fused image. Finally merged image is produced by replacing the intensity band of IHS data space with generated new intensity image and then converted to RGB data space by considering the previous Hue and Saturation bands. Pan sharpening scheme based on component substitution approach using the regression coefficients is proposed to merge the multispectral and PAN image [7]. In this approach first, the preliminary bands of the MS image such as red, green, blue and near infrared bands are transformed to the same spatial plan of PAN image. Then synthetic intensity image is obtained using the regression coefficients weighed sum of expanded bands of MS, further a detail component image is generated by subtracting the generated synthetic intensity image from PAN image. Next the yielding detail image is modulated using the second set of coefficients, then fused MS bands are obtained by adding modulated detailed image with all the expanded MS bands. Ultimately pan sharpened multispectral image is obtained by combining fused RGB bands. Pan sharpening method based on intensity-hue-saturation-brovey-transform with smoothing-filter intensity-modulation [IHS-BT-SFIM] approach is proposed to enhance the spatial details of multispectral image [8]. In this paper five different modules such as intensity-hue-saturation, intensity hue-saturation with brovey-saturation [IHS], brovey-transform [BT], brovey-transform with smoothing- filter- intensitymodulation [BTSFIM] and smoothing- filter- intensitymodulation [SFIM] are designed to present the frequency and ground details more accurately in a fused image. IHS module is merged with BT to solve the saturation problem that results a colour distortion. Further SFIM module is cascaded with IHSBT module to mitigate the colour distortion in fused image by providing more ground details. Hybrid pan sharpening technique based on injection of high frequency details into the various bands of multispectral image is proposed to enhance the ground details in fused image [9]. In this algorithm first, the difference image of PAN and intensity images is obtained, then high frequency components of this difference image is produced using Laplacian filtering. Then local gain coefficient is estimated using the standard deviation of multispectral bands and intensity image with local and global correlation coefficients of the same images. The computed local gain produced an edges of the ringed curved form because of geometric instabilities, hence the fusion parameter is post processed using Laplacian of Gaussian and selective mean filters to mitigate the local distortion issue of fusion parameter. Next obtained primary and secondary high frequency details are modified using the estimated local adaptive fusion parameters and combined with all the bands of multispectral image. The satellite image fusion scheme based on curvelet transform using ARISS theory is proposed to merge the MS and PAN image [10]. In this proposed method the curvelet transform is used as multi-scale model and inter-band structure model to fuse panchromatic and multispectral images. First complete spatial information are extracted from PAN and MS images using multi-scale model, then inter band structure model is used to infer the spatial information which is not there in the multispectral image. Finally, these missing local details extracted from the PAN image are inserted into MS image to obtain fused image with more ground details. In this proposed fusion scheme, a new algorithm is developed for pansharpening of multispectral image by integrating high spatial components of SAR and PAN images into multispectral image to enhance the perception a scene. This new algorithm is based on the combination of curvelet decomposition with selection fusion rule followed by gradient fusion. The selection fusion rule selects the specific details from synthetic aperture radar image to be merged with multispectral image.

Published By:

Eyes Intelligence Engineering Journal Website: www.ijeat.org

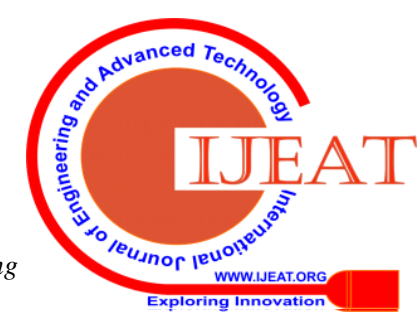


Generally features like building, bridges, road, etc. results a high backscattering response in the SAR image because of the high dielectric properties of some construction materials like metal and other objects. In particular SAR back scatter response is very high in the urban area where complex roof structures and different shapes high rise building are located seal jointly. Since man made features act as corner reflectors to SAR signals and complicated scattering mechanisms from the neighbouring building, irregularity results in the spatial details of the back scatter response [5]. Hence spatial pattern irregularities of urban areas in SAR image have heterogeneous characteristics, when SAR image is combined with multispectral image these irregularities in spatial domain results negative effect in fused image because of the difference of input images. In order to tackle this problem, synthetic aperture radar texture image is segmented into homogeneous and heterogeneous areas, then fusion excellence is improved by fusing these areas using curvelet decomposition with selection rule along with gradient fusion followed by hybrid pansharpening technique. The key goal of the proposed algorithm is to improve the local details of multispectral image by injecting high spatial details of SAR and panchromatic images into MS image. In this scheme first the panchromatic image spatial resolution is boosted by injecting the specific spatial details of SAR image into the PAN image. Then resulting high spatial information PAN-SAR (PS) fused image is used to pansharpening of multispectral image to enhance its spatial resolution with protecting the frequency information of the original multispectral image. The goal of the proposed algorithm is accomplished by using three stages of fusion scheme. The first stage of a fusion scheme used to perform pre-processing of SAR image, co registration of PAN, SAR and MS images, SAR feature extraction, decomposition of PAN and SAR texture images into fine and coarse coefficients plane using the curvelet transform. Next new fine coefficients of PAN image are obtained by inserting the specific fine coefficients of SAR image into fine coefficients of the panchromatic image using selective rule. Further an intermediate PAN image is obtained by applying inverse curvelet transform to new fine and unmodified coarse coefficients of panchromatic image. The first stage of a fusion scheme results a fused image which consists of nonadaptive sparse components of the SAR and PAN images having almost entire spatial details of SAR image. Next the second stage of fusion scheme uses the gradient fusion approach to obtain an additional information in the fused product. Finally, in the last stage hybrid pansharpening algorithm is proposed to sharpen the multispectral image. In this paper the proposed fusion scheme is systematized as follows. The fusion strategy is briefly described in the part II. Results and analysis carried out for various fusion methods and proposed method are examined in the part III. Inference and subsequent work are explained in the part IV.

\section{FUSION PROCEDURE}

The structure of the fusion procedure is described by using the eight different steps. In the first step, SAR preprocessing task used to diminish the inherent granular noise is carried out, the second step is exploited to find the pixel by pixel correspondence between the source images of the same area to align them geometrically. The third step is used to describe texture analysis process to obtain the homogeneity feature of SAR image, in the fourth step the process of segmenting the PAN and SAR images into coarse and fine coefficients using curvelet decomposition is carried out. The fifth step is used to inject the specific fine coefficients of SAR image into the fine coefficients of a decomposed PAN image, the six step is utilized to perform inverse curvelet transform to produce an intermediate PAN image. The seventh one is used to describe the procedure of gradient fusion to generate a PAN-SAR (PS) fused image. Finally, pansharpening of multispectral imaging procedure based on a hybrid pansharpening algorithm which injects high frequency information of the PS image into an original MS image to boost the spatial information while retaining the spectral details as much as possible is described. The figure 1 gives the flow diagram of the proposed fusion algorithm.

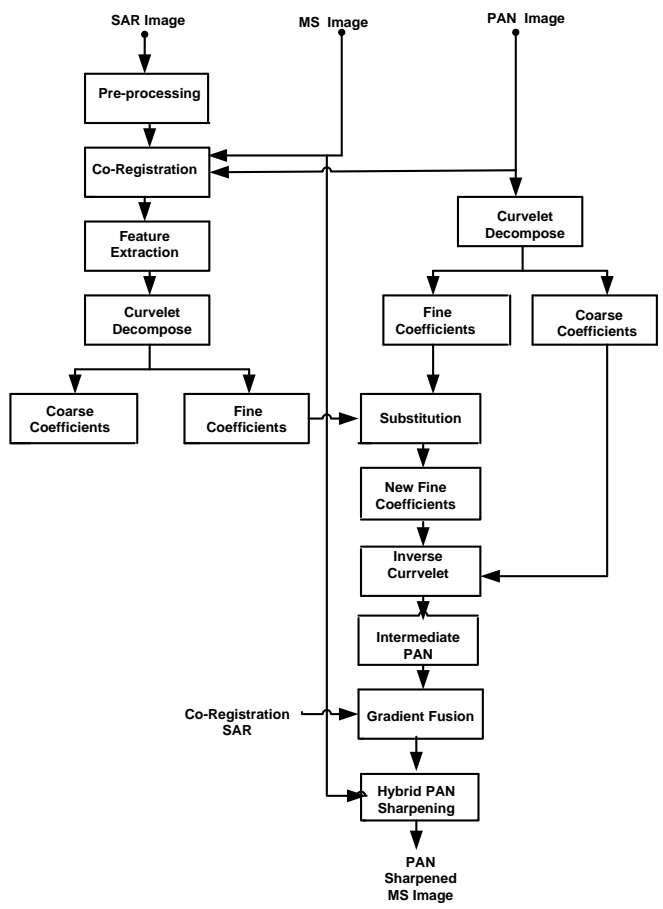

Figure 1: Flow diagram of the Proposed Fusion Scheme

\section{A. Preprocessing}

The multiplicative noise called speckle, inherently present in the SAR image because of radar signal complexity and scattering mechanism. In SAR image each single pixel consists of multiple scatters, which generates the speckles. These speckles humiliate the information present in the image along with producing the echoes, they also hamper with finding the precise location of objects in the image. The excellence of the fused image is openly affected by these speckles. Hence it is necessary to suppress the speckle noise before the image fusion process. In this paper, effective speckle noise removal filter is used to despeckle the SAR image with preserving the edge details [14]. This filter has three stages, in the first stage the block based modified PCA is used to mitigate the speckles in larger scale.

Blue Eyes Intelligence Engineering

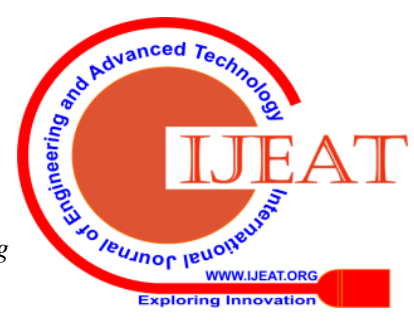

\& Sciences Publication

(C) Copyright: All rights reserved. 


\section{Fast Discrete Curvelet Decomposition with Gradient Fusion Based Technique for Pansharpening of Multispectral Image.}

In the filter second stage the residual multiplicative speckle noise present in the first stage output is eliminated using the hybrid median filter. The third stage uses the sharpening filter to improve the despeckle SAR image quality.

\section{B. Co-registration}

After despeckle of SAR image, the next step in any successful image fusion scheme is image registration. The fundamental step in the image fusion scheme is an image registration process, which estimates an optimal transformation to find the pixel by pixel correspondence between the source images of the same location. The image co-registration task aligns input images geometrically, which is the most important prerequisites and critical component of the image fusion process. In this paper snap tool is used to perform the image co-registration between the SAR, PAN and MS images which is fully an automatic and accurately co registered the SAR and MS images (slaves) with respect to the PAN image (master). In snap tool the used co-registration technique is based on cross correlation which provides an optimal geometric alignment between the slave and master images. This technique is segmented into the two parts, the first part is called coarse registration where the satellite orbits and timings are utilized to approximate the offsets or by identifying the approximate common points in the PAN (master) and SAR, MS (slaves) images and by carrying correlation matching with large windows. The second part is called fine registration which uses an automation correlation procedure to acquire accurate pixel by pixel alignment between the SAR, MS and PAN images. After computation of coarse and fine registration offsets, the co-registration polynomial are evaluated using warp operator which performs the resamples pixel of SAR , MS images into pixels of PAN image for geometric alignment. The image registration flow diagram is shown in figure. 2

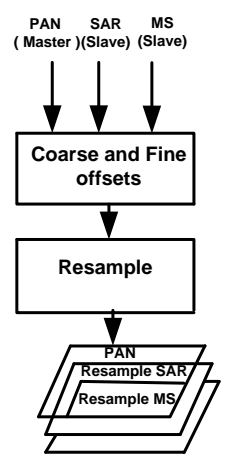

Figure 2: Image Registration Flow Diagram

\section{Feature selection}

In SAR image a high backscatter response results from manmade features such as high raised buildings, roads, bridges, etc., due to dielectric materials like alloy used to construct them and synthetic things geometric natures. The Synthetic radar backscatter response is extremely high in the more populated metropolitan city where large of number of high raised buildings, seal together with the multifaceted roof structure and different forms are located. Because of the more intricate synthetic radar signal and scattering mechanisms, buildings with complex structure, various shapes habitually act as corner reflectors which results the irregularities in the spatial pattern of the SAR image. Hence in the more populated metropolitan city, SAR image backscatter intensities contain mixed quality called heterogeneous characteristics. When SAR and PAN images are combined, due to the large difference between them, spectral distortion results. Because of heterogeneous characteristics, SAR images have an adverse effect in the fused product on the accountability of more populated metropolitan city where large of number of high raised buildings are located. Hence it is strict requirement to extract the heterogeneous characteristics from the SAR image before combining with PAN image. In this paper the snap tool is used to extract the heterogeneous characteristics of the SAR image using texture analysis called grey level co occurrence matrix (GLCM). GLCM computes the possibility of occurrence of two grey level separated in a given direction by a given distance. In any image, texture features represent the spatial information which are more valuable for image analysing tasks, like objects identification, classification, segmentation, etc.,. GLCM texture analysis process utilizes the inherent spatial information of the image and produces the several new images called texture features such as contrast, correlation, variance, homogeneity, dissimilarity etc.,. In this paper the homogeneity texture feature image which has high spatial information is selected for further image processing task.

\section{Curvelet decomposition}

The main drawback of wave transform (WT) is that which fails to smoothen the edges, this problem can be overcome by the curvelet transform (CT). The curvelet transform is a modified version of WT with aim of represent the objects in a nonadaptive manner with multi-scales and different angles. In this paper the curvelet transformation which has constructive geometric feature, is utilized for smooth representation of the local patterns present in an image by approximating the uniqueness of curvature parts with less coefficients. Wedge wrapping based fast discrete CT is applied for both SAR texture image and PAN to smoothen the local patterns in the image with different scales and angles.

\section{Wrapping Based Fast Discrete Curvelet Decomposition}

Wrapping based fast discrete curvelet decomposition (WFDCT) is one of the digital transform which returns sets of digital coefficients. These are indexed by scale, orientation and a spatial location parameter. This transformation is performed by considering $\mathrm{j}$ number of scale from finest to coarsest, 1 number of angles started from top left corner increases in clockwise with position $(k 1, k 2)$. In this paper the applied WDFCT generates fine and coarse digital coefficients by using the chain of translation and the wedge wrapping technique [21]. WDFCT is a linear transformation which takes Cartesian arrays as input in the form of $\mathrm{f}\left(\mathrm{x}_{1}, \mathrm{y}_{1}\right)$, for $0 \leq \mathrm{x}_{1}, \mathrm{y}_{1}<n$ for the image of size $n \times n$ and digital curvelet coefficients are computed by the using the equation (1).

Published By:

Retrieval Number: E9888069520/2020@BEIESP

Eyes Intelligence Engineering

\& Sciences Publication

(C) Copyright: All rights reserved.

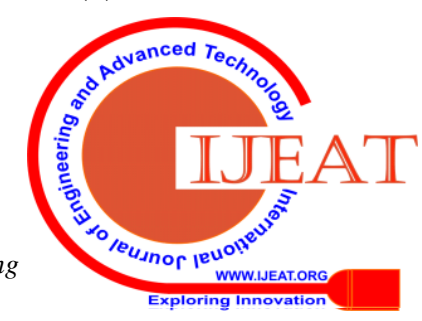


$C^{D}(j, k, l)=\sum_{0 \leq x 1, y 2 \leq n} f(\mathrm{x} 1, y 1) \overline{\varphi_{\jmath, l, k}^{D}(x 1, y 1)}$

where $\varphi_{j, l, k}^{D}$ is the digital curvelet waveform, D indicates digital, $\mathrm{f}(\mathrm{x}, \mathrm{y})$ digital image of size $n \times n$ with spatial variables $\mathrm{x}$ and $\mathrm{y}$, frequency domain variables $\omega_{1}, \omega_{2}$ with $\theta$ and $\mathrm{r}$ are polar coordinate in the spectral domain. Then radial and angular windows are computed by using equations (2) and (3) respectively, where $r \in(1 / 2,2)$ and $t \in(-1,1)$.

$\sum_{j=-\infty}^{\infty} w^{2}\left(2^{j} r\right)=1 \quad r \in\left(\frac{3}{4}, \frac{2}{4}\right)$

$\sum_{l=-\infty}^{\infty} v^{2}(t-l)=1$

$$
t \in\left(\frac{-1}{2}, \frac{1}{2}\right)
$$

where equation (2) represents the radial window and equation (3) represents angular window and these are positive, smooth and real valued. Next frequency domain window $U_{j}$ is produced using these two windows for $j \geq j_{0}$ by equation (4).

$U_{j}(r, \theta)=2^{\frac{-3 j}{4}} w\left(2^{-j} r\right) v\left(\frac{22^{\left|\frac{j}{2}\right|}}{2 \pi}\right)$

where $\frac{j}{2}$ 's the integer part is $\left|\frac{j}{2}\right|$, therefore support of frequency domain window is a polar wedge. It is define by angular and radial windows along with scale dependent widths in all the direction, applied to polar wedge. Consider waveform $\varphi_{j}(x)$ and its Fourier transform (FT) $\hat{\varphi}_{j}(\omega)$. Letting $U_{j}\left(\omega_{1} \omega_{2}\right)$ frequency window is equal to the $\hat{\varphi}_{j}\left(\omega_{1} \omega_{2}\right)$, where $\varphi_{j}$ mother curvelet which means the curvelets are defined at scale $2^{-j}$ generated by translations with rotation of $\varphi_{j}$. Where angle of rotation $\theta_{l}=2 \pi \times$ $2^{-|j / 2|} \times l, \quad l=0,1 \ldots \ldots . .$. where $0<\theta_{l}<2 \pi$ and rotation parameter sequence $k=\left(k_{1}, k_{2}\right)$. All these notations are used to define the curvelets at the scale $2^{-j}$, rotation angle $\theta_{l}$ with the position $\mathrm{x}_{k}^{(j, l)}=R_{\theta_{l}}^{-1}\left(\mathrm{k} 1 \times 2^{-j}, \mathrm{k} 2 \times 2^{-j / 2}\right.$ define by equation (5).

$\varphi_{j, k, l}(\mathrm{x})=\varphi_{j}\left(R_{\theta_{l}}\left(\mathrm{x}-\mathrm{x}_{k}^{(j, l)}\right)\right)$

$R_{\theta}$ is the orientation with radians $\theta$. Next inner product of the FT of image and frequency domain window is obtained. In this approach each rectangular spatial grid is translated into the curvelet at each scale with defined angle. Then by wrap the resulting product about the origin, the curvelet coefficients can generated. However, in this approach the frequency domain window $U_{j}\left(\omega_{1} \omega_{2}\right)$ is not fit into rectangular spatial grid of size $2^{j} \times 2^{j / 2}$ align with the axes where IFFT to be applied to determine fine and coarse curvelet coefficients. This problem could be conquer by defining the parallelogram $P_{j, l}$ containing the support of digital frequency window $U_{j}\left(\omega_{1} \omega_{2}\right)$. which has $L_{1, j} \sim 2^{j}$ and $L_{2, j} \sim 2^{j / 2}$ constants at each scale and for each rotation angle $\theta_{l}$. Two dimensional function can be overlay with the translates of parallelogram $P_{j, l}$ in both vertical and horizontal direction. The resulting windowed data is described by the equation (6)

$w d[n 1, n 2]=\sum_{m 1} \sum_{m 2} d\left[n 1+m 1 L_{1, j}, n 2+m 2 L_{2, j}\right]$

where windowed data $d\left(n_{1}, n_{2}\right)$ is equals to frequency domain window and FT of image inner product. Then obtained window data is wrapped the about the origin as constraint of $w d[n 1, n 2]$, indices $n 1, n 2$ inside the rectangular of size $L_{1, j} \times L_{2, j}$ near the origin, where $0 \leq n 1<L_{1, j}, 0 \leq n 2<L_{2, j}$. This window data can also be wrapped around the origin by using the modulo function define in the equation (7)

$W d\left[n 1 \bmod L_{1, j}, n 2+\bmod L_{2, j}=d[n 1, n 2]\right.$

where $(n 1, n 2) \in P_{j, l}$, this operation maps the window data to new position near the origin.

The WFCT is obtain as follows

- The Fourier samples of the given image is obtain by applying the FFT $\hat{f}(n 1, n 1) \frac{-n}{2}<n 1, \quad n 2<$ $\frac{n}{2}$

- Obtain the inner product of frequency domain window and fourier samples of an image for the all scales $\mathrm{j}$ and all the angles $\mathrm{l}$, i.e $\widetilde{U}_{j, l}(n 1, n 2) \hat{f}(n 1, n 1)$.

- Next wrapping the resulting product about the origin and get $\tilde{f}_{j, l}(n 1, n 2)=W\left(\widetilde{U}_{j, l} \hat{f}\right)[\mathrm{n} 1, \mathrm{n} 2]$, where $\mathrm{n} 1<\mathrm{L}_{1, \mathrm{j}}$ and $\mathrm{n} 2<\mathrm{L}_{2, \mathrm{j}}$ for $\theta(-\Pi / 4, \Pi / 4)$

- Since in this paper, two level scaling operation is performed, two different types of the digital curvelet coefficients such as fine coefficient $C^{D}(1, k, l)$ and coarse coefficient $C^{D}(2, k, l)$ are obtained by apply the IFFT to each $\tilde{f}_{j, l}$.

In the proposed fusion scheme texture feature extracted SAR and PAN images of size $512 \times 512$ are decomposed in two different scales and sixteen different orientations, which results the curvelets at two scale called fine and coarse. This gives the one approximate coefficients (fine) of size $341 \times 341$ and sixteen detailed coefficients of four of size $278 \times 342$, four of size $342 \times 278$, four of size $256 \times 342$ and four of size $342 \times 256$. The curvelet fine coefficients consists of low frequency details and detailed coefficients consists of high frequency with sixteen different orientations.

\section{E. Substitution}

After decompose of both PAN and texture feature extracted SAR image by applying two scale WFDCT, a new curvelet fine coefficients is produced combining SAR and PAN images by applying the block based feature selection rule. In this process SAR image curvelet fine coefficients are partially fused with fine coefficients of panchromatic, whereas the PAN coarse coefficients remain same. The technique used to produce the new PAN fine coefficients is denoted using equation (8).

$f_{N}(P S)=\left\{\begin{array}{lr}f_{N}(P) & \text { if } M(p)=\text { true } \\ f_{N}(P) \times \omega_{1}+f_{N}(S) \times \omega_{2} & \text { other wise }\end{array}\right.$

where $f_{N}(P S)$ is the a new PAN fine coefficients image, $f_{N}(P)$ is curvelet fine coefficients of PAN image and $f_{N}(S)$ is the curvelet fine coefficients of SAR image, $\mathrm{M}(\mathrm{p})$ is the block image, $\omega_{1}$ and $\omega_{2}$ are weight coefficients, computed using the standard deviation of SAR and PAN images are defined by using equations (9) and (10).

$$
\begin{aligned}
\omega_{1} & =\frac{\sigma_{S}}{\sigma_{P}+\sigma_{S}} \\
\omega_{2} & =\frac{\sigma_{P}}{\sigma_{P}+\sigma_{S}}
\end{aligned}
$$

Where $\sigma_{S}$ is the standard deviation of SAR image and $\sigma_{P}$ is the standard deviation of PAN image.

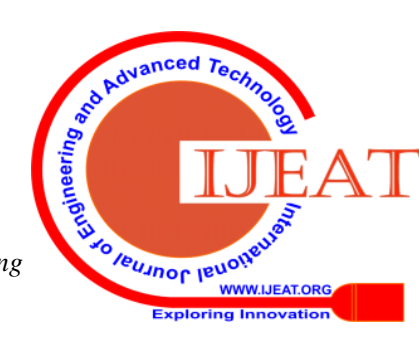

Blue Eyes Intelligence Engineering \& Sciences Publication

(C) Copyright: All rights reserved. 


\section{Fast Discrete Curvelet Decomposition with Gradient Fusion Based Technique for Pansharpening of Multispectral Image.}

Then an inverse WFDCT is applied to new curvelet fine coefficients and original PAN curvelet coarse coefficients image, to get an intermediate PAN image having the spatial distribution same as that of SAR image in homogeneous regions and in the rest of the parts, spatial details of original PAN image are retained with preserving nonadaptive sparse details of both the images.

\section{F. Gradient fusion}

In the second stage of the proposed system, the gradient fusion technique is used to portray information about the detail components present in both SAR and intermediate PAN images to which the human visual structure is more susceptible [15]. This approach, results the fused image which contains the more detail components than the SAR and PAN images along with preserving their original detail information. Gradient fused image is obtained as follows.

- For the both SAR and an intermediate PAN images, the gradient components are computed in both $\mathrm{x}$, $\mathrm{y}$ directions.

- Then gradient magnitudes are computed for both images using gradient components.

- Next the gradient magnitudes of both images are compared and the image having the maximum gradient magnitude at the each pixel location, that image pixel value is retained.

- From the resulting new image the gradient components are computed in $\mathrm{x}, \mathrm{y}$ directions to obtain fused gradient image.

Finally the SAR and PAN fused image is obtained by combining the gradient components of a fused gradient image using the wavelet based technique.

\section{G. Hybrid pansharpening of multispectral image}

The key goal of the any pansharpening algorithm is to provide the trade-off between the spectral and spatial information in the fused image. Several algorithms have been developed in the few decades to sharpen the multispectral image for different application by using local details of high spatial resolution panchromatic image. In one of the previous study, the hybrid pan-sharpening algorithm is proposed to sharpen the multispectral images by fusing the high spatial PS image obtained by using the wavelet decomposition to have a trade-off between spatial and spectral features in the fused image [5]. In this paper, a hybrid pansharpening algorithm is developed to inject both primary and secondary high frequency details of the PS image into multispectral images to improve spatial resolution. In this novel approach, the PAN image is replaced by the PS image which is obtained from using the two stage fusion scheme based on a curvelet decomposition with gradient fusion technique, which integrate the specific features SAR image into the PAN image [15]. The complete procedure of obtaining the high spatial information fused multispectral image bands [4], [5] is portrayed in equation (11).

PS_MS ${ }_{n}^{\mathrm{h}}=\mathrm{MS}_{\mathrm{n}}^{\mathrm{o}}+\lambda_{\mathrm{n}}\left[\delta_{\mathrm{n}} * \mathrm{H}_{\mathrm{n}}+\mu_{\mathrm{n}} * \mathrm{~L}\left(\mathrm{H}_{\mathrm{n}}\right)\right]$ where PS_MS ${ }_{n}$ is pansharpen high spatial $n^{\text {th }}$ band multispectral image, $\mathrm{MS}_{\mathrm{n}}^{\mathrm{o}}$ is an original $\mathrm{n}^{\text {th }}$ band multispectral image, $\lambda_{n}$ fusion parameter of $n^{\text {th }}$ MS band image, $H_{n}$ is high frequency information $\delta_{n}$ and $\mu_{n}$. are the adjustment coefficients and $\mathrm{L}$ is the Laplacian filter. The parameters used in the equation (11) are defined as follows [5],

$$
\lambda_{\mathrm{n}(\mathrm{x}, \mathrm{y})}=\sqrt{\frac{\sigma\left(\mathrm{MS}_{\mathrm{n}(\mathrm{x}, \mathrm{y})}^{\mathrm{o}}\right)}{\sigma\left(I_{(x, y)}^{l}\right)} S_{n(x, y)}}
$$

where $\lambda_{\mathrm{n}(\mathrm{x}, \mathrm{y})}$ is the local fusion parameter $S_{n}$ represents global correlation coefficient between the original $n^{\text {th }}$ band multispectral image $\mathrm{MS}_{\mathrm{n}}^{\mathrm{o}}$ and intensity image $I_{n}^{l}, \omega_{1}$ is weight coefficient used while injecting the specific texture features of SAR image into PAN image using curvelet decomposition, $\sigma($.$) is the standard deviation, and S_{n(x, y)}$ is the local correlation coefficient. The local standard deviation values of $\mathrm{MS}_{\mathrm{n}}^{\mathrm{o}}, I_{(n)}^{l}$ and $S_{n(x, y)}$ are computed using the local window size of 32x32. Intensity image $I_{(n)}^{l}$ is obtained by multiple regression between the each multispectral band and high resolution PS image PS ${ }^{\mathrm{h}}$. The adjustment coefficient $\mathrm{w} r \mathrm{t}$ various bands of the MS image is calculated using the equation (13)

$$
\delta_{\mathrm{n}}=\max \left[\frac{\sigma\left(I^{l}\right)}{\sigma\left(M S_{n)}^{o}\right.}, \frac{\sigma\left(M S_{n)}^{o}\right.}{\sigma\left(I^{l}\right)}\right]
$$

Primary high frequency components of the PS image $\mathrm{w} r \mathrm{t}$ to different MS bands are obtained by subtracting adjusted intensity image from high resolution histogram matched PS image and is show in equation

$$
\mathrm{H}_{\mathrm{n}}=\mathrm{PS}^{\mathrm{h}}-I_{n}^{l}
$$

The secondary high frequency components of the PS are obtained by performing Laplacian filtering of $\mathrm{H}_{n}$ which retains the fine details in the filtered image. The resulting filtered image is modified by the adjustment coefficients $\mu_{n}$ and is computed by using the equation shown below.

$$
\mu_{\mathrm{n}}=\frac{\sigma\left(H_{N}\right)}{2 \sigma\left(H_{n}^{\prime}\right)}
$$

where $\left(H_{n}^{\prime}\right)$ is Laplacian filtering of $\mathrm{H}_{\mathrm{n}}$

The whole pansharpening procedure can be concise as follows

i) Multiple regression coefficients corresponding $n^{\text {th }}$ band multispectral image is first computed

ii) The intensity image is generated using the RGB and infrared bands of MS [20] as follows

$$
\mathrm{I}^{\mathrm{l}}=\alpha_{0}+\sum_{n=1}^{N} \alpha_{n} M S_{n}^{o}
$$

where $\alpha_{n}$ regression coefficients corresponding $\mathrm{n}^{\text {th }}$ band MS image.

iii) The high resolution intensity image is produced with the help of MS and PS images using the equation shown below

$$
I_{n}^{h}=C C_{n} P S^{h}+\left(1-C C_{n}\right) M S_{n}^{o}
$$

iv) Next histogram matched PS image synthesized using the standard deviation and mean values of original MS and PS images as follows

$$
P S_{n}^{h}=P S^{h} \frac{\sigma\left(M S_{n}^{o}\right)}{\sigma\left(P S^{h}\right)}+\overline{M S_{n}^{o}}-\frac{\sigma\left(M S_{n}^{o}\right)}{\sigma\left(P S^{h}\right)} \overline{P S^{h}}
$$

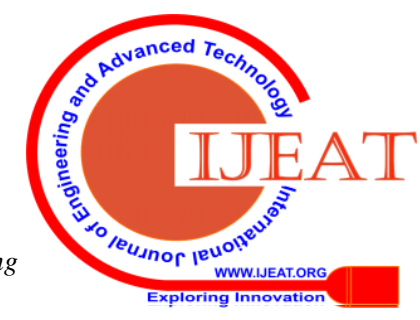



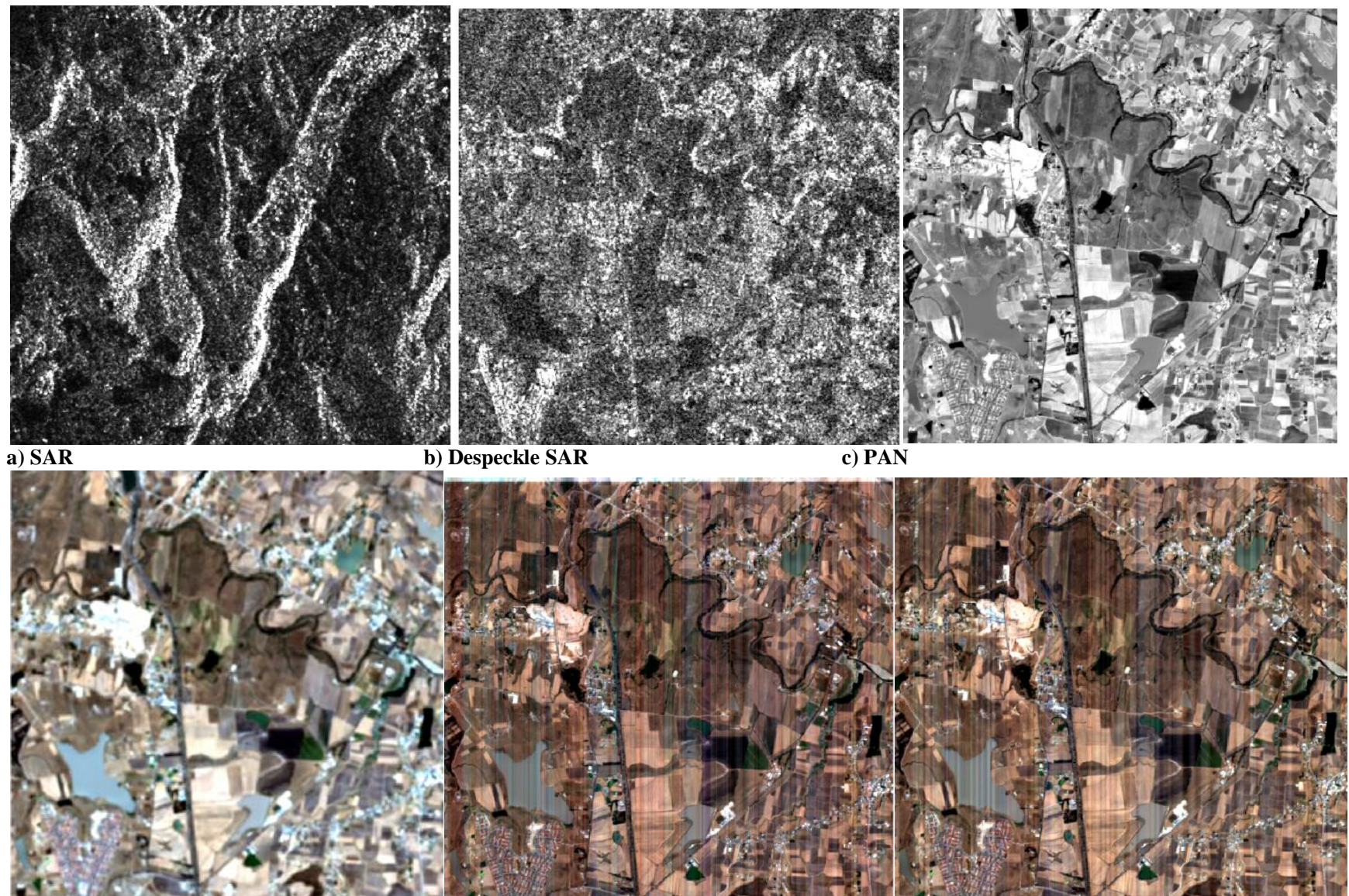

b) Despeckle SAR

c) PAN
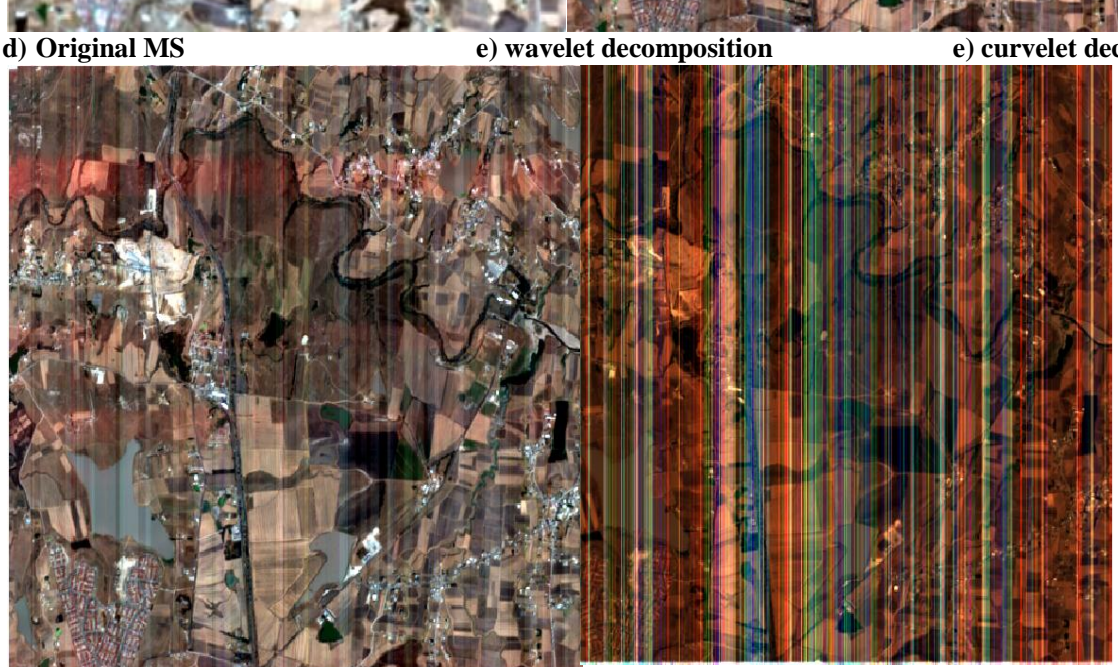

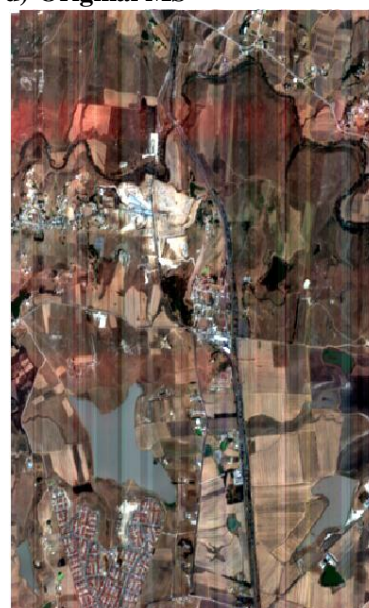

f) KL transform

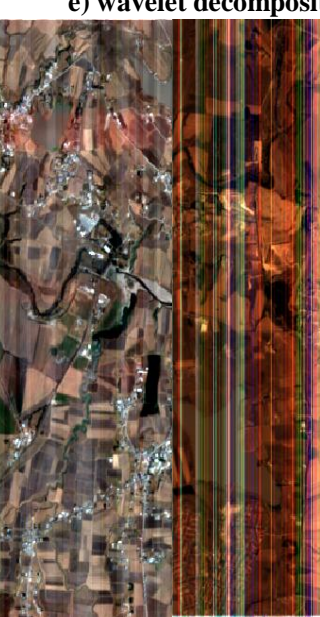

g) PC fusion
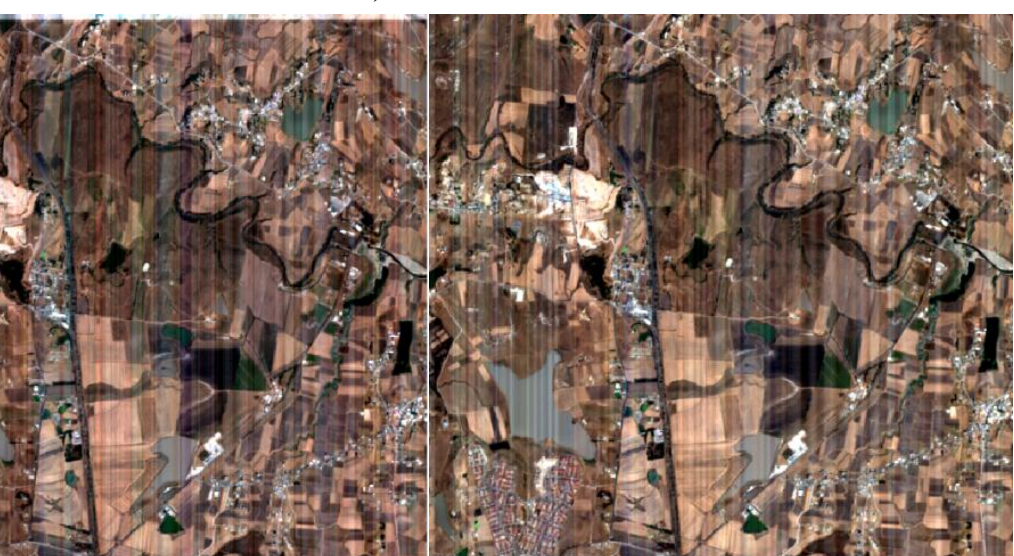

e) curvelet decomposition

Figure.3: MS pansharpened images a) SAR, b) Despeckle SAR, c) PAN, d) Original MS, e) Wavelet Decomposition f) Curvelet Decomposition, g) KL transform , h) PC fusion and i) Proposed Method.

v) The proposed pansharpening algorithm depends on the a vector $\lambda_{\mathrm{n}(\mathrm{x}, \mathrm{y})}$ (fusion parameter) corresponding to each band of MS which applied to the complete band of MS. However, due to the different image formation mechanism dissimilarity in spatial domain occurs between SAR and MS images, the fusion parameter cannot produce an optimal fused image since this parameter in not able to reflect the spatial details of MS image. To obtain the local details of source images, the local fusion parameter is computed using a local standard deviation and mean values of original MS and adjusted intensity images along with global, local correlation coefficients using equation (12). Next because of numerical uncertainty, a looped curve shaped edge appears in the resulting $\lambda_{\mathrm{n}(\mathrm{x}, \mathrm{y})}$. These edges are eliminated by applying using Laplacian of Gaussian filter. Then through this local fusion parameter, the spatial clarity and spectral preservation of source images can be control and improve. Meantime, the proposed fusion algorithm is optimized to pansharpening of multispectral image using the equation (11).

\section{RESULTS AND ANALYSIS}

In this paper SAR and multispectral images obtained from sentinel 1 and sentinel 2 sensors are used to evaluate the performance of the proposed fusion algorithm. The study location used for assessing the proposed fusion scheme situated in Mexico, North America.

Published By:

Blue Eyes Intelligence Engineering \& Sciences Publication 


\section{Fast Discrete Curvelet Decomposition with Gradient Fusion Based Technique for Pansharpening of Multispectral Image.}

This selected region is suburban area consists of roads, vegetation, ponds and buildings. SAR image was captured on February 08, 2019 and multispectral image was captured on February 06, 2019. The sentinel 2 sensor consists of thirteen spectral bands varying from ten to sixty meter resolution. Among these thirteen spectral bands, four bands like red, green, blue and near infrared have ten meter resolution. The panchromatic image is generated by averaging the values of red, green, blue and near infrared bands to have more spatial information. Before the merging process, from both the images the overlapping regions with same size are cropped to obtain the same scene using the operation called subset in snap toolbox. Also multispectral image data sets are resampled to make their spatial resolution same as SAR and that of panchromatic image. Table I shows the sentinel 1 and sentinel 2 data specifications. Qualitative and quantitative evaluations carried out to assess the proposed fusion algorithm by comparing with the fused image produced from existing methods. To evaluate the fused image performance obtain by integrating SAR, MS, and PAN images quantitatively, different spatial as well as spectral metrics are computed for various existing methods and proposed method. The estimated spectral metrics were the relative dimensionless global error in synthesis (ERGAS), the spectral angle mapper (SAM), the relative average spectral error (RASE) [12], [16] and $\mathrm{Q}_{\mathrm{avg},}$, [11], [13].

Table I Sentinel 1 and 2 data Specifications

\begin{tabular}{|c|c|c|}
\hline Parameter & Sentinel 1 data & Sentinel 2 data \\
\hline Acquisition Date & $08 / 02 / 2019$ & $06 / 02 / 2019$ \\
\hline Image size & $512 \times 512$ & $512 \times 512$ \\
\hline Spatial Resolution & $\begin{array}{c}3.5 \mathrm{~m} \text { (pixel } \\
\text { spacing) (SAR) }\end{array}$ & $\begin{array}{c}10 \text { m (pixel spacing) } \\
\text { (PAN) }\end{array}$ \\
\hline Product Type & GRD & S2_MSI_LEVEL_2A \\
\hline
\end{tabular}

The lesser the values of metrics SAM, RASE, ERGAS and higher the value of the metric $\mathrm{Q}_{\mathrm{avg}}$ indicate that spectral similarities between the original MS and pansharpened MS images is better [17]. Meantime, root mean square error (RMSE), spatial correlation coefficients (SCC), structural similarity index (SSIM) between the original MS and pansharpened images were computed to assess the spatial clarity and sharpness of the fused image. The estimator SCC gives the spatial similarity between the fused and PS images, higher the value of SCC, SSIM and lower the value of RMSE indicate that better the spatial similarity between the fused and PAN images.

Table II Performance metrics of various Pansharpening methods

\begin{tabular}{|l|l|l|l|l|l|l|l|}
\hline $\begin{array}{l}\text { Pansharpening } \\
\text { Method using }\end{array}$ & SAM & ERGAS & RASE & QAVG & SSC & SSIM & RMSE \\
\hline Wavelet & 2.478 & 0.5292 & 32.8820 & 0.6765 & 0.726 & 0.939 & 1.140 \\
\hline Curvelet & 2.0717 & 0.4563 & 28.1741 & 0.7396 & 0.776 & 0.949 & 0.977 \\
\hline KL Transform & 2.154 & 0.6355 & 40.7930 & 0.6258 & 0.687 & 0.917 & 1.415 \\
\hline PC Fusion & 14.47 & 2.7221 & 155.019 & 0.1633 & 0.289 & 0.244 & 5.377 \\
\hline Proposed & $\mathbf{0 . 3 9 2}$ & $\mathbf{0 . 1 0 2 1}$ & $\mathbf{6 . 4 5 7 9}$ & $\mathbf{0 . 9 8 3 1}$ & $\mathbf{0 . 9 8 4}$ & $\mathbf{0 . 9 9 6}$ & $\mathbf{0 . 2 2 4}$ \\
\hline
\end{tabular}

These metrics were computed for various pansharpening algorithms such as pansharpening using wavelet decomposition, curvelet decomposition, KL transform, PC fusion method, Brovey method and proposed fusion scheme.Figure.3 illustrates the MS pansharpened images results from various existing algorithms and proposed algorithm. Table II indicates the values of the estimation metrics of the pansharpened MS image obtained from various algorithms. As table II shows, the proposed algorithm results the excellent values for all the spectral and spatial indices. This excellent performance of proposed algorithm in spatial domain is mainly due to the injection of specific feature of high spatial resolution SAR image into panchromatic image using curvelet decomposition followed by gradient fusion technique. The Lower values of spectral indexes like SAR, ERGAS,RASE results from the injection of primary and secondary high frequency components into the MS image. Hence the proposed fusion algorithm preserves the almost complete spectral information of original MS image having spatial characteristics of SAR image in the fused image

\section{CONCLUSION}

In this work, a novel approach based fusion algorithm is proposed for the multisensory image fusion where block based wrap fast discrete curvelet decomposition, gradient fusion technique and hybrid pansharpening algorithm were employed to address the fusion of SAR, MS and PAN

Published By:

Retrieval Number: E9888069520/2020@BEIESP

Blue Eyes Intelligence Engineering Journal Website: www.ijeat.org

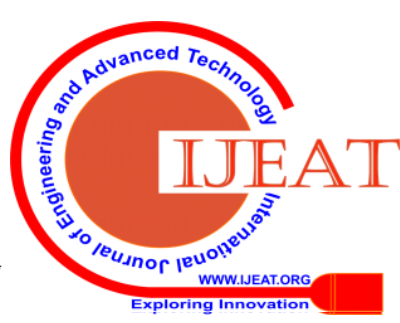


images. In the first section of the proposed work, multisensor images of the same area are aligned geometrically, then the SAR texture image is segmented into homogeneous and heterogeneous areas.

Further homogeneous areas of SAR image merged with PAN image using block based curvelet decomposition and the pixel based selection rule and heterogeneous areas are merged using only PAN details. This process shows that an intermediate PAN image as well as the final fused image interpretability can be effectively increased by incorporating only the selected SAR texture feature such as homogeneous areas into PAN image. The proposed fusion algorithm is verified using SAR and multispectral images obtained from sentinel 1 and sentinel 2 sensors. To test the performance, the proposed algorithm is evaluated using both visual and quantitative and compared the various other fusion methods. The evaluated result shows that the proposed algorithm performance is better than the other techniques, because almost all fused image results from other techniques exhibit the colour distortion with less spatial resolution. However the proposed fusion algorithm can provide, the more spatial details in the fused image without any colour distortion, since it can provide information based on the selection rule with curvelet decomposition which takes the area characteristics into account. The focused subsequent work will be on mapping and interpretation process using fused obtained from the proposed algorithm rather than those of individual images.

\section{REFERENCE}

1. Jie Hu, Zhi He, and JieminWu, " Deep Self-Learning Network for Adaptive Pansharpening " Remote Sensing Journal, Vol 11, pp 2395, May 2019.

2. Yong Yang1, Weiguo Wan1, Shuying Huang2, Feiniu Yuan1, Shouyuan Yang1, and Yue Que1, " Remote Sensing Image Fusion Based on Adaptive IHS and Multi-scale Guided Filter ", IEEE Access Remote Sensing Image Fusion Journal, Volume 4, pp 4573 -4582, 2016.

3. Mufit Cetin and Abdulkadir Tepecik, " Intensity Hue Saturation Applied Remote Sensing, Vol. 10, pp 045019-1 to15, Oct-Dec 2016 ,

4. Jaewan Choi, Dongyeob Han and Yongil Kim, " Context-Adaptive Pansharpening Algorithm for High-Resolution Satellite Imagery ", Canadian Journal of Remote Sensing, Vol. 38, No. 1, pp. 109_124, 2012.

5. Younggi Byun, Jaewan Choi, and Youkyung Han, " An Area- Based Image Fusion Scheme for the Integration of SAR and Optical Satellite Imagery, " IEEE Journal Applied Earth Observations and Remote Sensing, Vol.6, No. 5,pp 2212-2219, October 2013.

6. Myungjin Choi, " A New Intensity-Hue-Saturation Fusion Approach to Image Fusion With a Trade off Parameter ", IEEE Transactions On Geo-science and Remote Sensing, Vol. 44, No. 6, pp 1672-1682, June 2006.

7. Bruno Aiazzi, Stefano Baronti, Member, IEEE, and Massimo Selva , " Improving Component Substitution Pansharpening Through Multivariate Regression of MS+Pan Data", IEEE Transactions on Geoscience and Remote Sensing, VOL. 45, NO. 10, pp 3230- 39, OCT 2007.

8. Te-Ming Tu, Ching-Luh Hsu, Pin-Yi Tu and Ching-Hai Lee, " An Adjustable Pan-Sharpening Approach for IKONOS/QuickBird/GeoEye-1/WorldView-2 Imagery ", IEEE Journal of Applied Earth Observations and Remote Sensing, Vol 5, No. 1, pp 125-133, Feb 2012.

9. Jaewan Choi, Junho Yeom, Anjin Chang, Younggi Byun, and Yongil Kim, " Hybrid Pansharpening Algorithm for High Spatial Resolution Satellite Imagery to Improve Spatial Quality ", IEEE Geo-science and Remote Sensing Letters, Vol. 10, No. 3, pp 490-494, May 2013. based image fusion using iterative linear regression ", Journal of

10. Myungjin Choi, Rae Young Kim, Myeong-Ryong Nam, and Hong Oh Kim, " Fusion of Multispectral and Panchromatic Satellite Images Using the Curvelet Transform ", IEEE Geoscience and Remote Sensing Letters, Vol. 2, No. 2, pp 136-140, Apr 2005.

11. Amina Jameel, Muhammad Mohsin Riaz, and Abdul Ghafoor, " Guided Filter and IHS based Pan-Sharpening ", IEEE Sensors Journal, Vol 7, No 4, pp 1530-437, 2015.

12. Yadong Song,WeiWu, Zheng Liu, Xiaomin Yang, Kai Liu, and WeiLu, " An Adaptive Pansharpening Method by Using Weighted Least Squares Filter ", IEEE Geo-science and Remote Sensing Letters, pp 1-5, 2015.

13. Shuyuan Yang, Min Wang, Licheng Jiao, " Fusion of multispectral and panchromatic images based on support value transform and adaptive principal component analysis ", Elsevier, pp 177-184, 2010.

14. Leelavathi H P and Dr. J Prakash, " Effective Speckle Noise Removal of SAR Image Based on Combination of Modified PCA and HMF with Enhancement " , International Journal of Engineering Trends and Technolog, Vol 61, No 3, July 2018.

15. Leelavathi H P and Dr. J Prakash, " Two Stage Fusion Scheme Based on a Curvelet with Gradient Technique for Integration of SAR Image on PAN Image to Improved the Spatial Resolution ", International Journal of Advanced Science and Technology, Vol. 29, No. 5, pp. 4041 - 4053, Apr 2020.

16. Luciano Alparone, Stefano Baronti, Andrea Garzelli and Filippo Nencini, "A Global Quality Measurement of Pan-Sharpened Multispectral Imagery ", IEEE Geo-science and Remote Sensing Letters, Vol. 1, No. 4, Oct 2004.

17. Luciano Alparone, Bruno Aiazzi, Stefano Baronti, Andrea Garzelli, Filippo Nencini, and Massimo Selva, " Multispectral and Panchromatic Data Fusion Assessment Without Reference ", Photogrammetric Engineering \& Remote Sensing , Vol. 74, No. 2, pp 193-200, Feb 2008.

18. Andrea Garzelli, Filippo Nencini and Luca Capobianco, " Optimal MMSE Pan Sharpening of Very High Resolution Multispectral Images " , IEEE Transactions On Geo-science and Remote Sensing, Vol. 46, No. 1, pp 228-236, Jan 2008.

19. Amr M. Ragheb - Heba Osman - Alaa M. Abbas, "Simultaneous Fusion and Denoising of Panchromatic and Multispectral Satellite Images", Sensing and Imaging An International Journal, Springer, Dec 2012.

20. Sheida Rahmani, Melissa Strait, Daria Merkurjev, Michael Moeller, and Todd Wittman, " An Adaptive IHS Pan-Sharpening Method ", IEEE Geo-science And Remote Sensing Letters, Vol. 7, No. 4, Oct 2010.

21. Emmauel Candes, Laurent Demanet, David Donoho and Lexing Ying," Fast Discrete Curvet Transforms,"applied and Computational Mathematics, Caltec, Pasadena, Stanfor, CA, July 2005, revised March 2006.

\section{AUTHORS PROFILE}

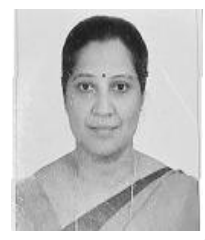

Leelavathi H P received the B.E degree in Electronics and Communication Engineering from Mysore University, Karnataka, India, in 1996 and M.Tech degree in Digital Communication and Engineering from VTU 2009. she is presently working as Associate Professor in the department of Electronics and Communication and Engineering, Vivekananda Institute of Technology, Bangalore. She is currently pursuing the Ph.D degree in VTU in the area of image processing.

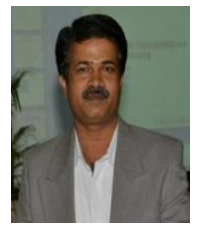

Dr. J. Prakash received the B.E degree in Computer Science and Engineering from Mysore University, Karnataka, India, in 1989, M.S degree in IT from University of Indore, Madhya Pradesh, India in 1995 and Ph.D from Dr. MGR Educational and Research Institute, University, Chennai, India in 2010. From 1989 to 1996 he worked as lecturer in the department of Computer Science and Engineering at Adichunchanagiri Institute of Technology, Chikmagalur, Karnataka, India.

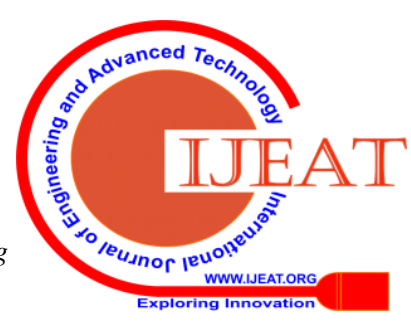


Fast Discrete Curvelet Decomposition with Gradient Fusion Based Technique for Pansharpening of Multispectral Image.

From 1997 to 2010 he worked as Assistant Professor of Information Science and Engineering, at Bangalore Institute of Technology, Bangalore, Karnataka, India. Since 2010, He is working as Professor and Head of Information Science and Engineering, Bangalore Institute of Technology, Bangalore, Karnataka, India. His research area of interest is Digital Signal Processing and Digital Image Processing with applications. Dr. J. Prakash is a Executive council member of Indian Society for Technical Education, New Delhi, India. 